\title{
Edge State in Pipe Flow Experiments
}

\author{
A. de Lozar, ${ }^{1,2, *}$ F. Mellibovsky, ${ }^{3}$ M. Avila, ${ }^{1,4}$ and B. $\operatorname{Hof}^{1, \dagger}$ \\ ${ }^{1}$ Max Planck Institute for Dynamics and Self-Organization, 37073 Göttingen, Germany \\ ${ }^{2}$ Max Planck Institute for Meteorology, 20146 Hamburg, Germany \\ ${ }^{3}$ Universitat Politècnica de Catalunya, 08034 Barcelona, Spain \\ ${ }^{4}$ Institute of Fluid Mechanics, Friedrich-Alexander-Universität Erlangen-Nürnberg, 91058 Erlangen, Germany
}

(Received 27 October 2011; published 21 May 2012)

\begin{abstract}
Recent numerical studies suggest that in pipe and related shear flows, the region of phase space separating laminar from turbulent motion is organized by a chaotic attractor, called an edge state, which mediates the transition process. We here confirm the existence of the edge state in laboratory experiments. We observe that it governs the dynamics during the decay of turbulence underlining its potential relevance for turbulence control. In addition we unveil two unstable traveling wave solutions underlying the experimental flow fields. This observation corroborates earlier suggestions that unstable solutions organize turbulence and its stability border.
\end{abstract}

DOI: 10.1103/PhysRevLett.108.214502

PACS numbers: 47.27.Cn, 47.27.N-, 47.52.+j

In most situations of practical interest fluid flows are turbulent. Often transition to turbulence occurs despite the linear stability of the laminar state [1,2] such as in flows through pipes, ducts or even in astrophysical Keplerian flows. In some other cases turbulence occurs well below the critical point given by linear instability analysis, such as in flows through channels. Moreover, it has been shown for these shear flows that the turbulent state has unstable characteristics [3-7] and that localized turbulent patches eventually decay back to laminar. That at higher Reynolds numbers turbulence is still the rule rather than the exception is due to its invasive nature which causes laminar gaps to be quickly consumed by adjacent turbulent domains [8,9]. The observation that localized turbulent domains are intrinsically unstable $[3,4,10,11]$ offers prospects to control and relaminarize flows [12]. Such potential methods are of great practical interest because the drag in turbulent flows is significantly larger and this causes higher energy consumption and limits transport rates.

From a dynamical point of view the stability boundary separating laminar from turbulent motion plays a key role in how flows transit to and from turbulence. This laminarturbulent boundary is highly convoluted and most likely possesses a fractal structure as shown in simulations [13]. Some signatures of this have also been observed in experiments [14]. Hence its complexity puts a complete description for transition in shear flows beyond reach in the foreseeable future. However, using a tracking method first proposed and applied to plane Poiseuille flow [15,16], it has been possible to compute phase-space trajectories on the laminar-turbulent boundary of pipe flow $[17,18]$. Surprisingly, the dynamics at this boundary, or edge, are organized by a single state: This so-called "edge state" [13] is a chaotic attractor within the edge, whereas in the full phase-space it is a repeller with a single unstable direction pointing towards turbulence on one side and towards laminar flow on the other.

According to dynamical systems theory the disordered dynamics of turbulence as well as of its edge are organized around unstable solutions of the Navier-Stokes equations [19]. For pipe flow mainly traveling wave solutions have been identified $[1,20,21]$ and simulations have shown the existence of at least two traveling waves embedded in the edge state [22]. The purpose of the present Letter is to explore if such dynamical systems concepts also play a role in practical situations. In particular we aim to determine if the edge state is relevant to control and relaminarization of turbulence.

In order to study the turbulent-laminar boundary in an experiment we apply a control method, which as recently shown, leads to relaminarization of turbulent structures at moderate Reynolds numbers [12]. Experiments were carried out in a $12 \mathrm{~m}(400 \mathrm{D})$ long pipe made of 1 metre sections with an inner diameter of $D=30 \pm 0.01 \mathrm{~mm}$. The working fluid, water in our case, enters the pipe from a reservoir using gravity as the driving force. Before each experiment the Reynolds number is adjusted to a value of $1900 \pm 10$. In pipe flow $\operatorname{Re}=U D / \nu$, where $U$ is the mean flow velocity and $\nu$ the kinematic viscosity. The continuous perturbation is generated by a syringe pump that simultaneously injects and extracts the exact same amount of fluid through a couple of mutually facing holes. A relatively large perturbation amplitude was chosen (1\% of the mean flow in the pipe) and the flow becomes turbulent directly at the perturbation point. Downstream of this point the flow is highly turbulent and for distances larger than $10 D$ the turbulence intensity starts to drop and then gradually goes to zero over the next $40 D$. The velocity vector field is measured in two observation planes in the decaying regime, one $27 \mathrm{D}$ and the other $33 \mathrm{D}$ downstream from the perturbation. Measurement planes 
are perpendicular to the pipe axis and all three velocity components within the planes are recorded using a (Lavision $\mathrm{GmbH}$ ) high-speed stereo PIV system (one for each plane). Because of the fast downstream advection structures change little while they move over short distances (order of $1 D$ ). To a first approximation the spatial structure can be recovered from the temporally resolved measurement by multiplication with the mean advection speed of the flow structures (Taylor's frozen turbulence hypothesis). This speed is obtained from the velocity twoplanes correlation function (see supplemental material (SM) Fig. 1, [23]).

An example of a velocity field sampled in decaying turbulence is shown in Fig. 1(a). Here velocities were averaged over $t=1.1 \mathrm{~s}$ which based on the mean velocity corresponds to a spatial average over 3.1D. Regions slower than the parabolic laminar flow are shown in blue and faster ones in red. The central part is dominated by a low-speed streak (blue) which is positioned offcenter and flanked by two high-speed streaks (red). These streaks are generated by streamwise vortices [see velocity vectors in Fig. 1(a)] located between the high and low-speed regions. This flow structure closely resembles the edge state [Fig. 1(b)], which so far had only been observed in numerical simulations [17,24]. Further investigations of the experimental data show that flow fields are mildly chaotic with the dominant frequency corresponding to a length of $3.1 D$. Two instantaneous snapshots spaced by $1.55 D$ are shown in Figs. 1(c) and 1(e). While in the first a strong streamwise vortex is located between the low-speed streak and the lower high-speed streak, in the second snapshot a strong streamwise eddy is now above the low-speed streak. Such streak-vortex dynamics are typical of traveling wave solutions $[20,21,25,26]$ and indicate that the flow may be dynamically close to one of them. The corresponding snapshots for the numerical edge state calculated for the same parameter values are shown in Figs. 1(d) and 1(f) and identical structural features can be observed. Edge state simulations were carried out in a short periodic pipe of $L=\pi D$ using the shoot and refine method described in $[17,18]$ and an accurate pipe flow code based on the solenoidal Petrov-Galerkin formulation presented in [27]. The spectral resolution was set to $33 \times 77 \times$ 27 axial $\times$ azimuthal $\times$ radial grid points. To establish how frequently such close correspondence between the experiment and the edge state occurs, we selected eight instances of the edge simulations representative of the mildly chaotic variations in its flow structure. These were then correlated with the experimental data. Following an identification method proposed for finding traveling wave transients in numerical simulations of turbulence [28] we defined two correlation functions: one that accounts for all velocity components, $I_{\text {tot }}$, and another, $I_{\mathrm{uv}}$, that is computed using solely the in-plane

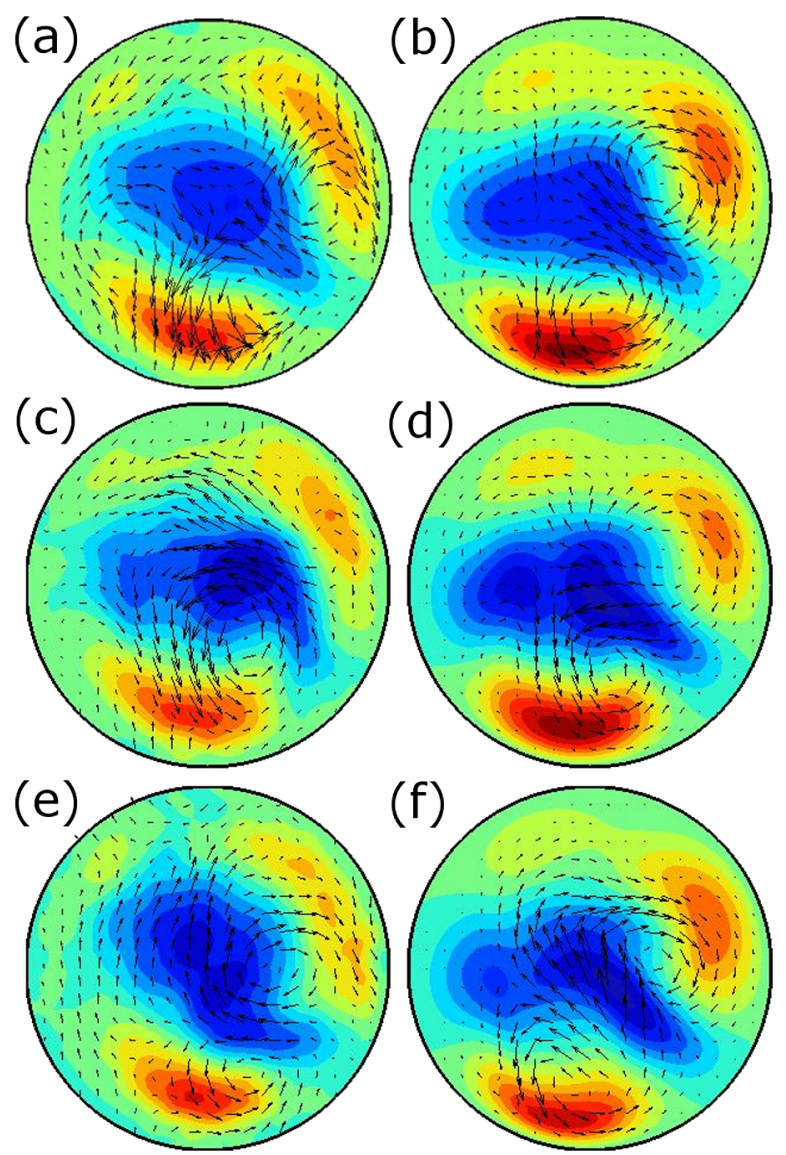

FIG. 1 (color online). Comparison of experimental and numerical cross-sectional velocity fields. (a) Experimental measurement averaged over $\pi D$ in decaying turbulence. (b) Snapshot of the numerical edge state averaged over the numerical domain. Instantaneous cross sections of the experimental and numerical edge state are shown in (c),(e) and (d),(f), respectively. The instantaneous cross sections are spaced by $(\pi / 2) D$. In-plane velocity components are indicated by arrows, while axial velocity is color coded (blue for negative, red for positive). The laminar parabolic profile has been subtracted. Color ranges from $-0.6 U$ to $0.6 U$ in (a)-(b) and from $-0.7 U$ to $0.7 U$ in (c)-(f), in steps of $0.1 U$. Maximum in-plane velocities in (a)-(b), and (c)-(f) are $0.07 U$ and $0.11 U$ respectively. See SM Video 1, [23] for a more detailed comparison.

(azimuthal and radial) velocity components. As proposed in [28] for values of $I_{\text {tot }}>\lambda$ and $\left(I_{\mathrm{uv}}+I_{\mathrm{tot}}\right)>2 \lambda$, with $\lambda=0.5$ the structural resemblance is very high so that such cases can be regarded as a visit to the particular state in question (here the edge state). Following this procedure we observe that for decaying turbulence the edge state is visited during $17 \%$ of the experimental time (see SM Fig. 2, [23]). While this is a considerable fraction of time, the true value is likely to be even larger. First because measurement noise reduces correlations and second because the experimental data is obtained at a fixed axial location only. Hence crossings of the turbulent-laminar boundary which take place slightly up- or downstream are not registered. 
Further evidence of the dynamical similarity between decaying turbulence and the edge state can be obtained by a phase-space projection as the one shown in Fig. 2, depicting 3D energy $\left(\varepsilon_{3 \mathrm{D}}\right)$ against pressure gradient $\left((\boldsymbol{\nabla} p)_{z}\right)$. The experimental trajectories (orange) have been calculated from the reconstruction of the velocity fields in a pipe of length $\pi D$ for each considered time instant. The chaotic excursions of the experimental trajectory are clearly confined to the area spanned by the numerical edge state (shown in blue) and are well separated from the part of the phase space where turbulence resides (red).

A characteristic feature of the edge state is that it is organized around specific unstable solutions. In particular an asymmetric traveling wave ( $S 1$ in the naming convention of [29]) and a mirror-symmetric traveling wave (M1) have been found to be embedded in the edge state [22]. To more stringently test the connection between the decaying turbulence and the edge state we tested if traveling waves could also be identified starting from experimental data. In order to do so, one approximate wavelength of the experiment was fed into a Newton-Raphson method [30] after suitable preprocessing of the experimental velocity field. This involved a conversion of a period of the time-resolved experimental velocity field into a full wavelength using Taylor's frozen turbulence hypothesis, followed by adequate smoothing and accurate projection onto the solenoidal periodic basis of the numerics. It should be noted that convergence to traveling wave

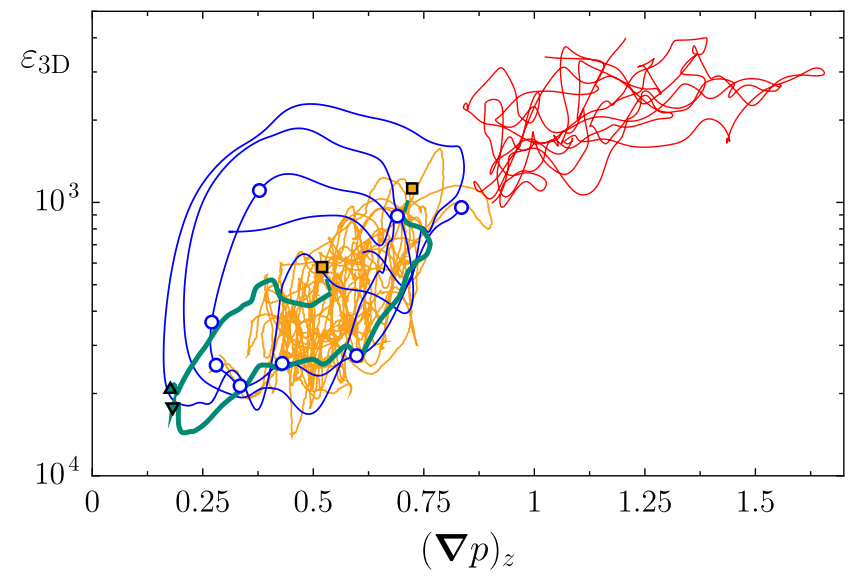

FIG. 2 (color online). Energy contained in streamwisedependent modes (3D Energy, $\varepsilon_{3 \mathrm{D}}$ ) vs driving pressure gradient $\left[(\nabla p)_{z}\right]$. Represented are the numerical edge state evolution (blue), a numerical turbulent run (red) and the experimental results obtained from measurements of decaying turbulence (orange). The down-pointing triangle indicates the asymmetric shift-reflect traveling wave solution $(S 1)$ while the up-pointing triangle represents the shift-reflect mirror-symmetric wave $(M 1)$ from which $S 1$ bifurcates [31]. The numerical edge state samples used for correlation are indicated with open circles. The squares indicate two close visits, the upper right corresponding to Fig. 1. The green lines illustrate convergence from experimental fields to $S 1$ and $M 1$ via Newton iteration. solutions had so far only been achieved from computed velocity fields and even then the method only succeeded for very close initial guesses. As shown in Fig. 2 (lower green line) the experimental initial condition indeed converged to $S 1$. The same procedure was repeated using a different experimental snapshot as initial condition. This second initial condition converged to $M 1$, as indicated by the upper green line in Fig. 2. The three dimensional structure of the flow fields before and after Newton convergence is shown in Fig. 3. The overall arrangement of the intertwined vortical structures closely agree. Before convergence, structures are broader and have an unsteady component. The Newton method smoothly transfers this velocity field to the underlying traveling wave solution. The convergence of the velocity fields in decaying turbulence confirms earlier suggestions that such unstable solutions provide the building blocks of fluid flow turbulence $[25,26]$. While close resemblance between coherent structures in turbulence experiments and exact unstable solutions had been reported, the present study provides further evidence of the connection and supports it quantitatively.

To probe the distance of our decaying turbulence to the actual laminar-turbulent boundary we initiated the numerical edge tracking method with the same preprocessed experimental data set as used for the Newton scheme. Only a small adjustment of around 6\% in amplitude was required to place the experimental flow field on the laminar-turbulent boundary. Furthermore, as shown in (a)

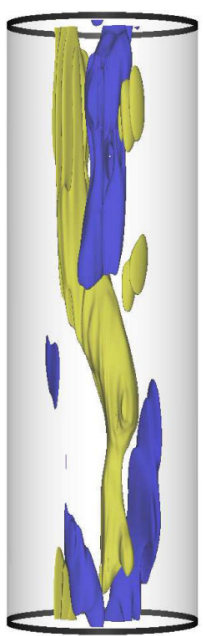

(b)

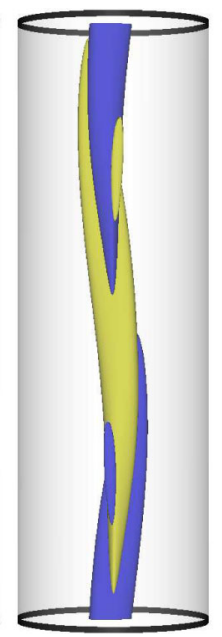

(c)

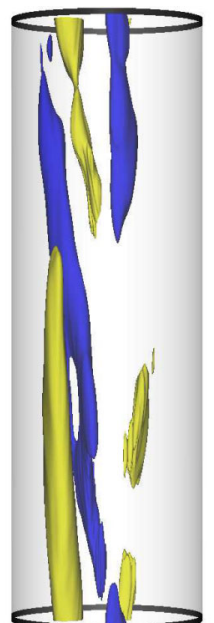

(d)

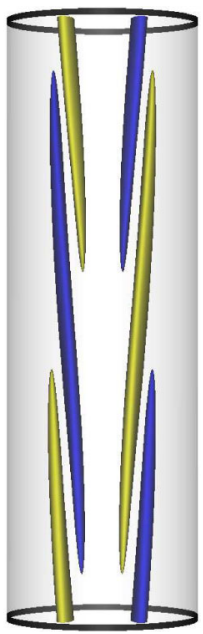

FIG. 3 (color online). Axial vorticity isosurfaces of (a) the experimental snapshot of Fig. 1 and (b) the traveling wave converged as a result of seeding the experimental snapshot to the Newton iteration. A second experimental snapshot is shown in (c), together with the traveling wave converged from it in (d). The isosurfaces correspond to $\omega_{z}= \pm 0.8 U / D$ (a), $\pm 0.4 U / D$ (b) and $\pm 0.5 U / D$ (c and $\mathrm{d}$ ), respectively. Blue for negative, yellow for positive. Fluid flows from top to bottom. 


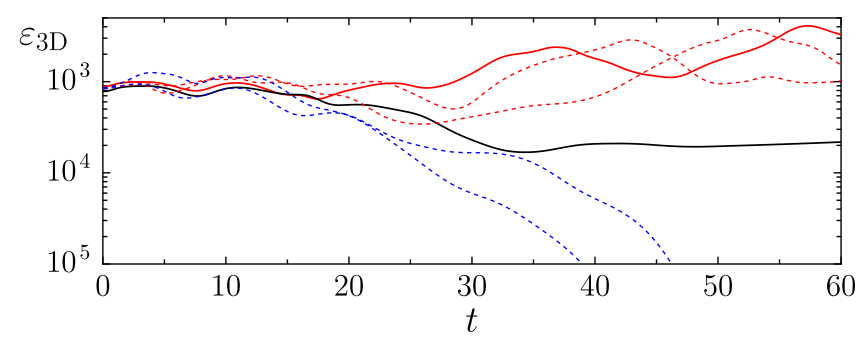

FIG. 4 (color online). Numerical time evolution starting from the experimental snapshot of Fig. 1 (solid red) and from four other experimental snapshots corresponding to shifts of 0.57 and $1.14 D$ upstream (dashed red) and downstream (dashed blue). The solid black line corresponds to a run from the same experimental initial condition but this time confined to the laminarturbulent boundary by applying an edge tracking method. The close resemblance of the evolution for over $15 \mathrm{D} / U$ confirms that the initial conditions are close to the edge state.

Fig. 4, the time evolution started from the experimental field (red solid line) and the edge tracking (black solid line), evolve together for over $15 \mathrm{D} / U$ time units (over 7 wavelengths of $S 1$ ) before the former parts from the latter and goes turbulent. In order to further substantiate the proximity of the experimental realization of the edge to the actual boundary, we started numerical evolution from experimental fields corresponding to 0.57 and $1.14 D$ upstream (dashed red) and downstream (dashed blue) shifts of the original experimental snapshot. Adjacent initial conditions have a split probability of relaminarizing or transitioning to turbulence, indicating that the flow is indeed marginally close to the edge state.

Finally we compared the advection speed of the coherent structures in decaying turbulence to that of the numerical edge as well as to the phase velocity of the $S 1$ and $M 1$ solutions. In the experiment the phase velocity is obtained from the correlation between the velocity fields measured in two different planes. We define the two-planes correlation function as: $c_{p 1 p 2}(d t)=\sum_{\text {visits }} \int \vec{u}_{p 1}(t) \cdot \vec{u}_{p 2}(t+$ $d t) d V$, where $\vec{u}$ is the in-plane velocity field reconstructed in a volume of length $3.1 \mathrm{D}$ and the summation goes over all approaches to the numerical edge state as defined above (17\% of the experimental time). The two-planes correlation function presents a distinct maximum at $d t=$ $4.11 \mathrm{D} / \mathrm{U}$. This corresponds to an advection velocity of $U_{\text {exp }}=1.46$. In comparison the phase velocity of the traveling waves with the same wavelength is $U_{S 1}=1.525$ and $U_{M 1}=1.502$. The advection velocity of the numerical edge state, has a mean value of $\bar{U}_{\text {edge }}=1.46$ and hence exactly matches the experimental value.

In summary we have verified the existence of the edge state in a laboratory experiment. The dynamics displayed during the relaminarization of turbulence follows the laminar-turbulent border previously only predicted in numerical simulations. The convergence of an experimental velocity field to nontrivial solutions of the Navier
Stokes equations substantiates recent claims that such solutions provide the building blocks of turbulence. This methodology will allow us in the future to search for unstable solutions underlying coherent structures commonly observed in turbulence experiments at moderate Reynolds numbers. We speculate that a better understanding of the edge state and its stable and unstable manifolds may play a crucial role in developing efficient turbulence control schemes.

Support from the Max Planck Society, the Engineering Physical Sciences Research Council (Grant No. EP/ F017413/2) and the Deutsche Forschungsgemeinschaft (project FOR 1182) is gratefully acknowledged.

*adelozar@googlemail.com

†bhof@gwdg.de

[1] B. Eckhardt, T. M. Schneider, B. Hof, and J. Westerweel, Annu. Rev. Fluid Mech. 39, 447 (2007).

[2] A. Meseguer and L. N. Trefethen, J. Comput. Phys. 186, 178 (2003).

[3] B. Hof, J. Westerweel, T. M. Schneider, and B. Eckhardt, Nature (London) 443, 59 (2006).

[4] B. Hof, A. De Lozar, D. J. Kuik, and J. Westerweel, Phys. Rev. Lett. 101, 214501 (2008).

[5] A. de Lozar and B. Hof, arXiv:1001.2481v1.

[6] D. Borrero-Echeverry, M. Schatz, and R. Tagg, Phys. Rev. E 81, 025301 (2010).

[7] E. Rempel, G. Lesur, and M. Proctor, Phys. Rev. Lett. 105, 044501 (2010).

[8] K. Avila, D. Moxey, A. deLozar, M. Avila, D. Barkley, and B. Hof, Science 333, 192 (2011).

[9] D. Barkley, Phys. Rev. E 84, 016309 (2011).

[10] A. de Lozar and B. Hof, Phil. Trans. R. Soc. A 367, 589 (2009).

[11] M. Avila, A. Willis, and B. Hof, J. Fluid Mech. 646, 127 (2010).

[12] B. Hof, A. De Lozar, M. Avila, X. Tu, and T. Schneider, Science 327, 1491 (2010).

[13] J. D. Skufca, J. A. Yorke, and B. Eckhardt, Phys. Rev. Lett. 96, 174101 (2006).

[14] Y. Tasaka, T. M. Schneider, and T. Mullin, Phys. Rev. Lett. 105, 174502 (2010).

[15] T. Itano and S. Toh, J. Phys. Soc. Jpn. 70, 703 (2001).

[16] S. Toh and T. Itano, J. Fluid Mech. 524, 249 (2005).

[17] T. M. Schneider, B. Eckhardt, and J. A. Yorke, Phys. Rev. Lett. 99, 034502 (2007).

[18] F. Mellibovsky, A. Meseguer, T.M. Schneider, and B. Eckhardt, Phys. Rev. Lett. 103, 054502 (2009).

[19] G. Kawahara, M. Uhlmann, and L. van Veen, Annu. Rev. Fluid Mech. 44, 203 (2012).

[20] H. Faisst and B. Eckhardt, Phys. Rev. Lett. 91, 224502 (2003).

[21] H. Wedin and R. Kerswell, J. Fluid Mech. 508, 333 (2004).

[22] Y. Duguet, A. P. Willis, and R. R. Kerswell, J. Fluid Mech. 613, 255 (2008). 
[23] See Supplemental Material at http://link.aps.org/ supplemental/10.1103/PhysRevLett.108.214502 for details.

[24] T. M. Schneider and B. Eckhardt, Phil. Trans. R. Soc. A 367, 577 (2009).

[25] B. Hof, C. van Doorne, J. Westerweel, F. Nieuwstadt, H. Faisst, B. Eckhardt, H. Wedin, R. Kerswell, and F. Waleffe, Science 305, 1594 (2004).

[26] B. Hof, C.W.H. van Doorne, J. Westerweel, and F. T. M. Nieuwstadt, Phys. Rev. Lett. 95, 214502 (2005).
[27] A. Meseguer and F. Mellibovsky, Applied Numerical Mathematics 57, 920 (2007).

[28] R. R. Kerswell and O. R. Tutty, J. Fluid Mech. 584, 69 (2007).

[29] C. C. T. Pringle, Y. Duguet, and R. R. Kerswell, Phil. Trans. R. Soc. A 367, 457 (2009).

[30] F. Mellibovsky and B. Eckhardt, J. Fluid Mech. 670, 96 (2011).

[31] C. C. T. Pringle and R. R. Kerswell, Phys. Rev. Lett. 99, 074502 (2007). 